CERN-TH-5720/90

\title{
3D MAXWELL-CHERN-SIMONS SPONTANEOUS SYMMETRY BREAKING
}

\author{
C. Aragone \\ CERN - Geneva \\ and \\ Departamento de Fisica, Universidad S. Bolivar \\ Apartado 8900, Caracas 1080A, Venezuela \\ and \\ P. Arias \\ Departamento de Fisica, Universidad S. Bolivar \\ Apartado 8900, Caracas 1080A, Venezuela
}

\begin{abstract}
We study the spontaneous symmetry breaking of the $3 D$ Maxwell-Chern-Simons (MCS) system. This action has one transverse, massive, parity sensitive degree of freedom. We show that after symmetry breaking, this excitation remains massive (although with an increased value) while the other transverse, parity sensitive, vector-like degree of freedom of the system (initially non-excited) acquires mass. These two masses are always different. Covariant, light front and canonical analysis of the massive MCS system are given as well as the two coupled reduced actions. The quartic behaviour of the system propagator stems in this coupled structure.
\end{abstract}

CERN-TH-5720/90

April 1990 


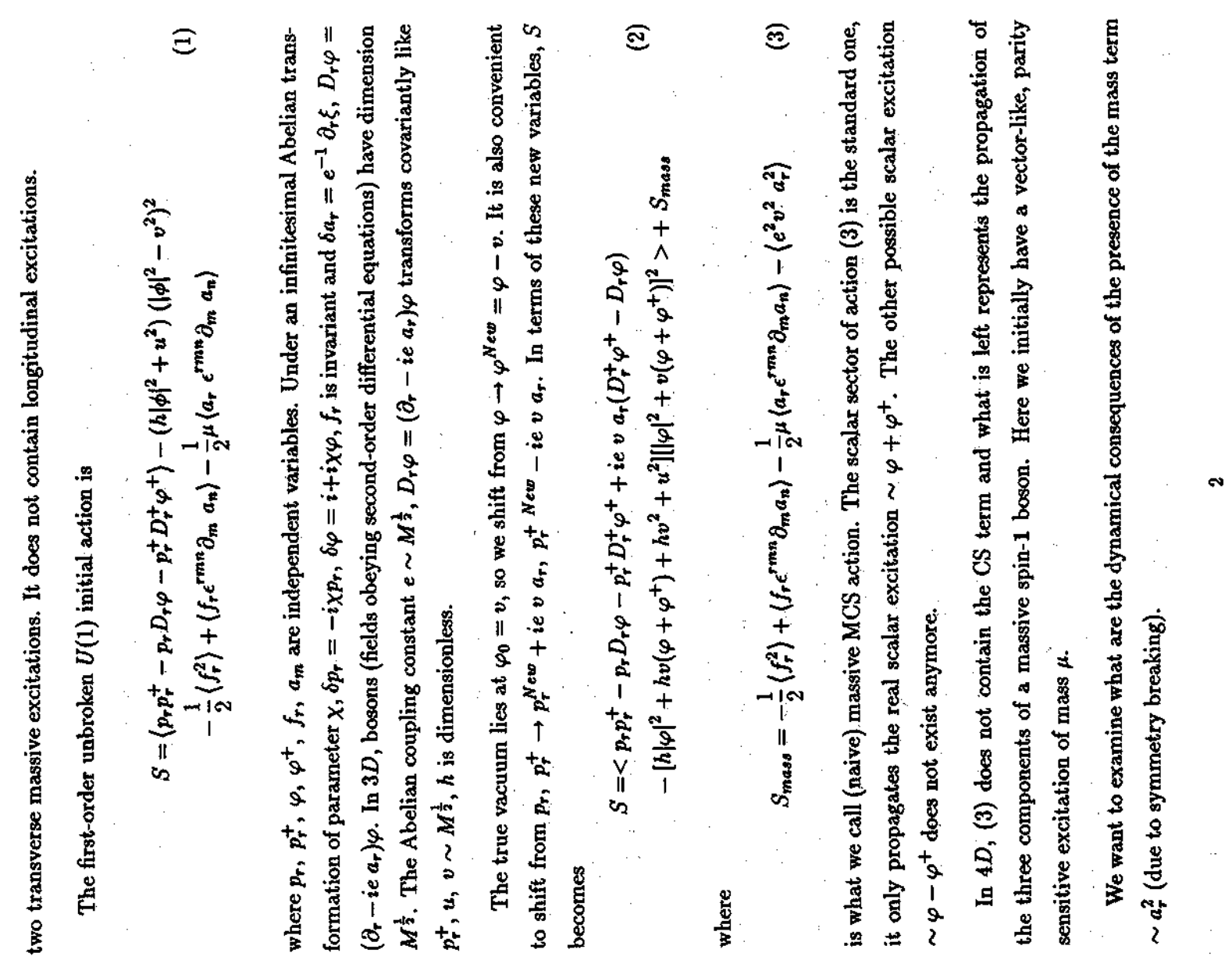

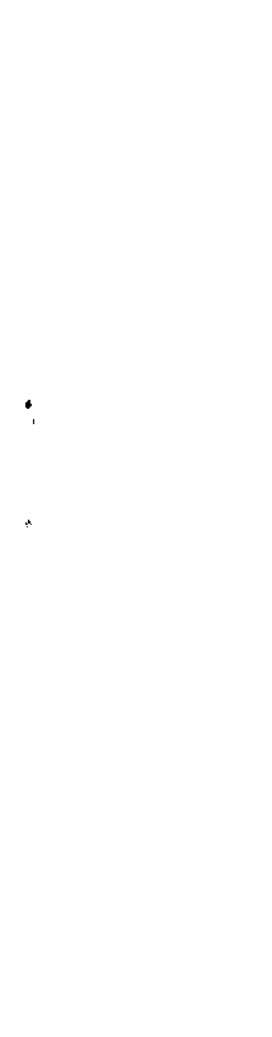

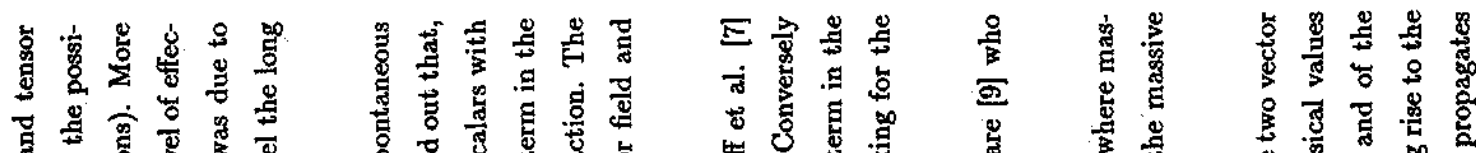

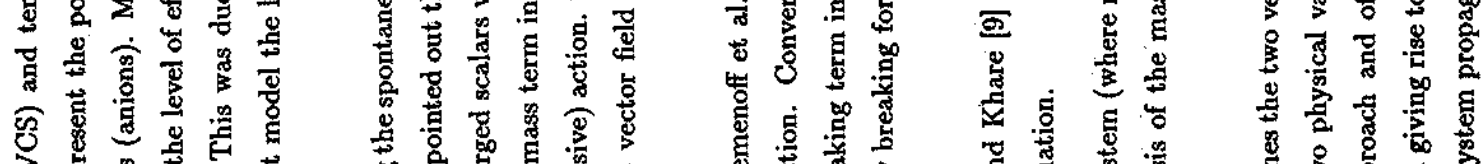

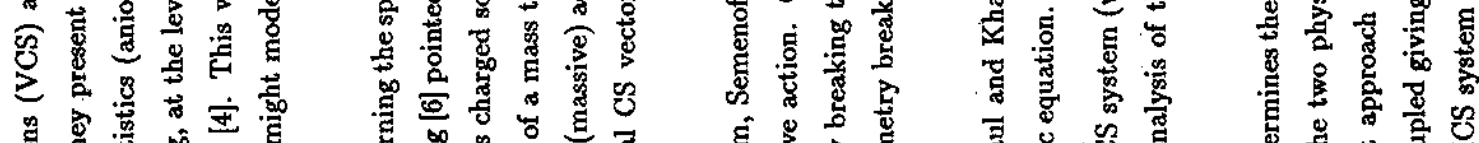

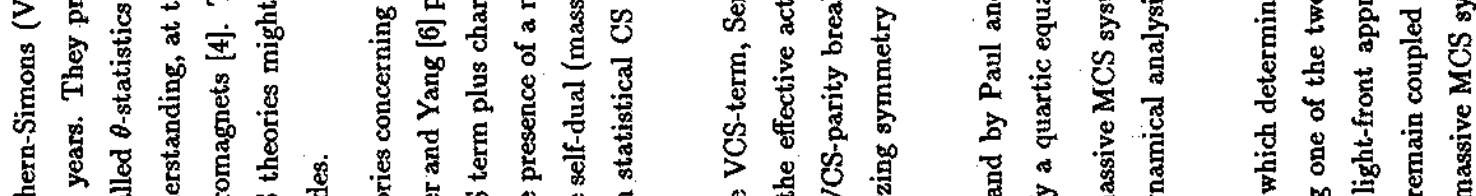

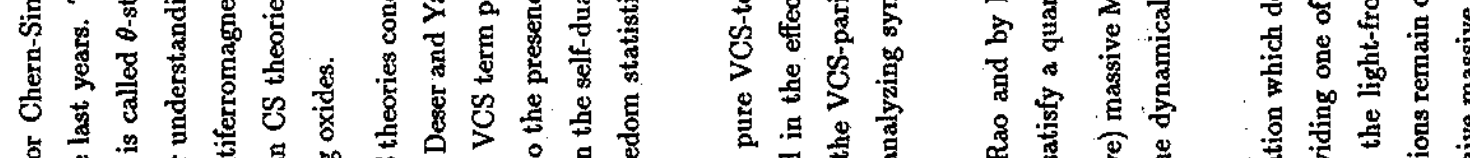

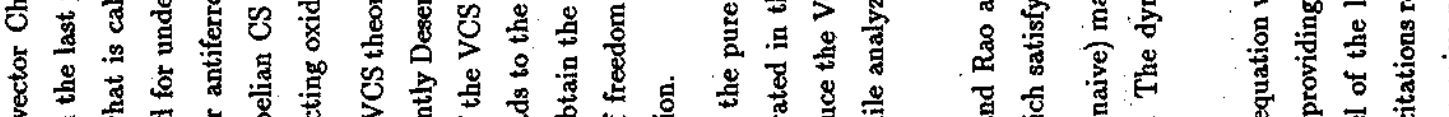

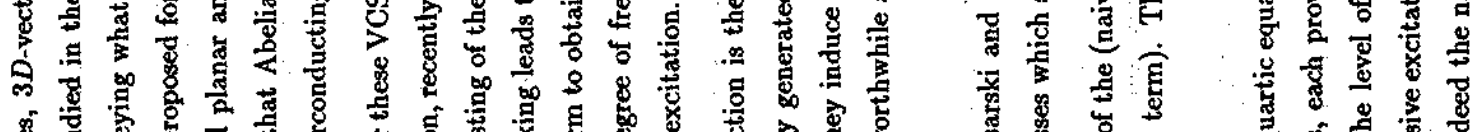

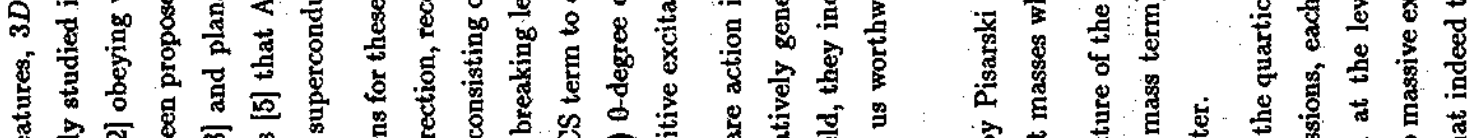

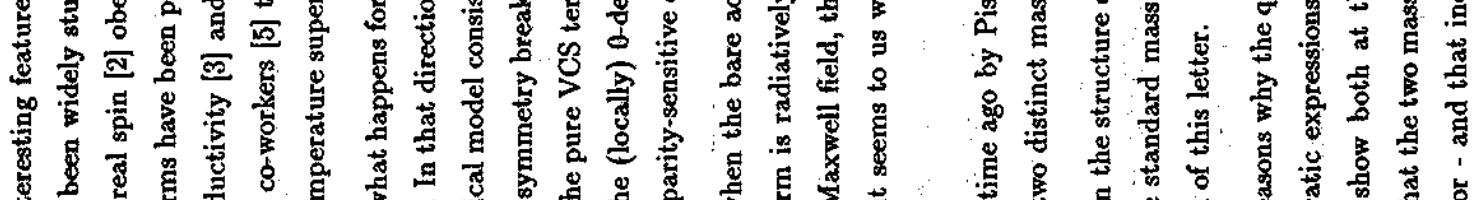

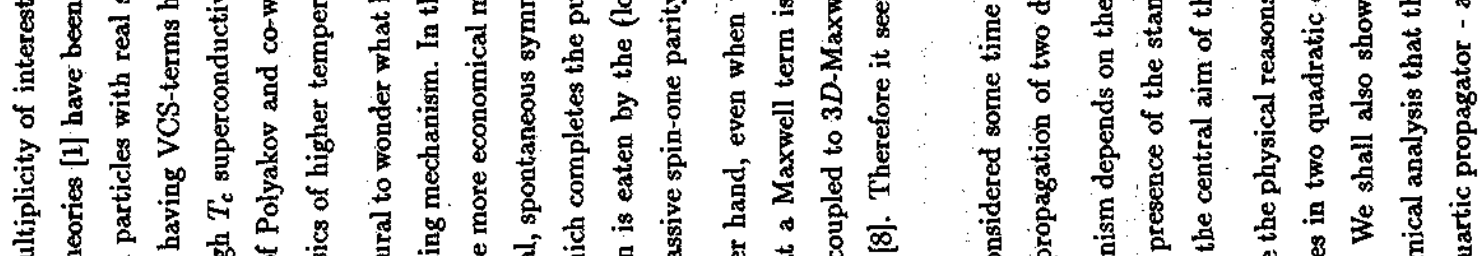

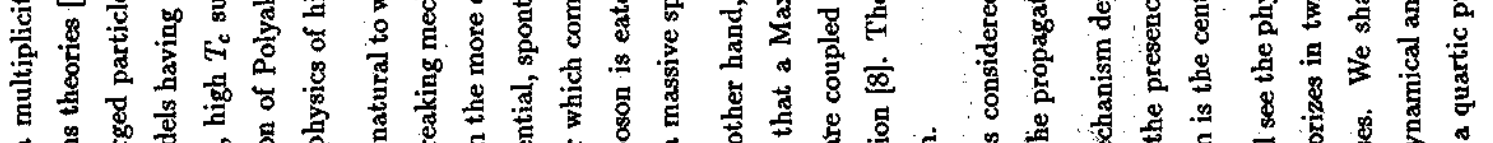

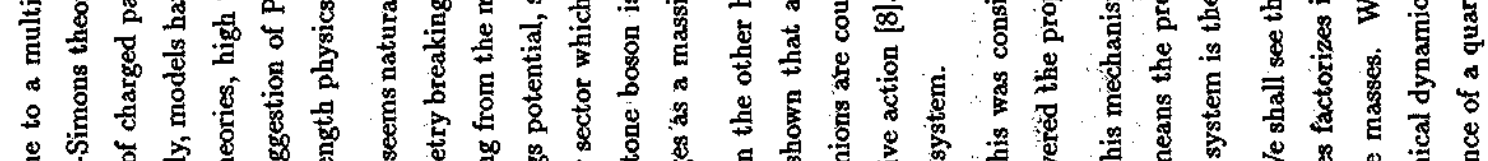

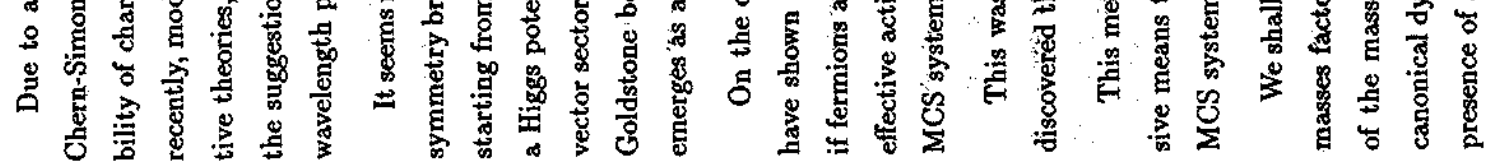


$\Xi$ ฐ

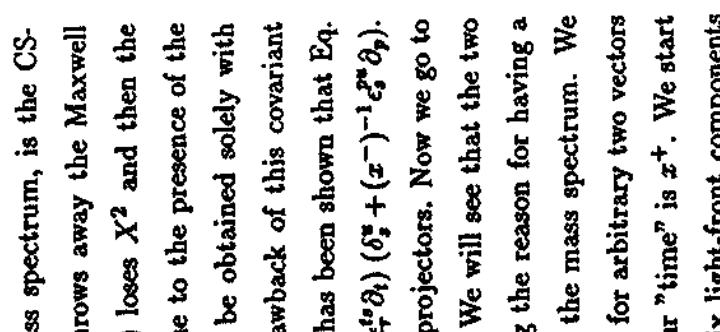

종

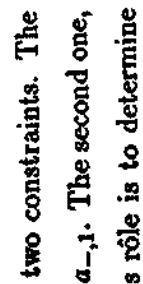

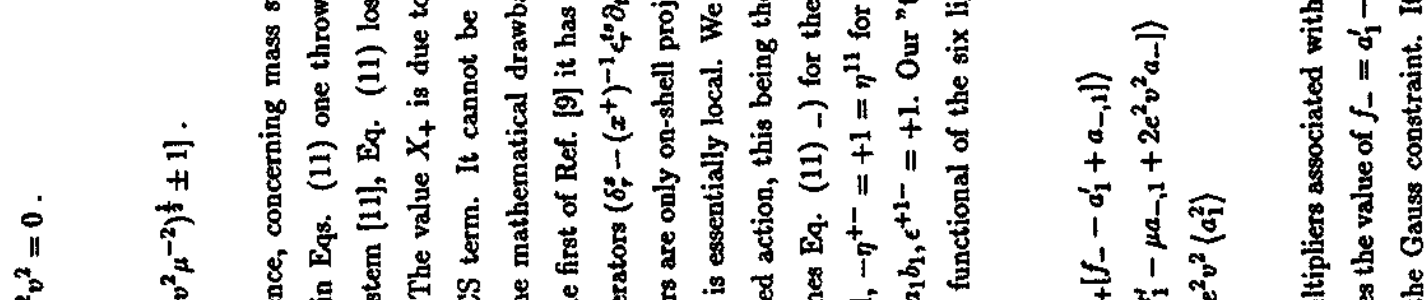

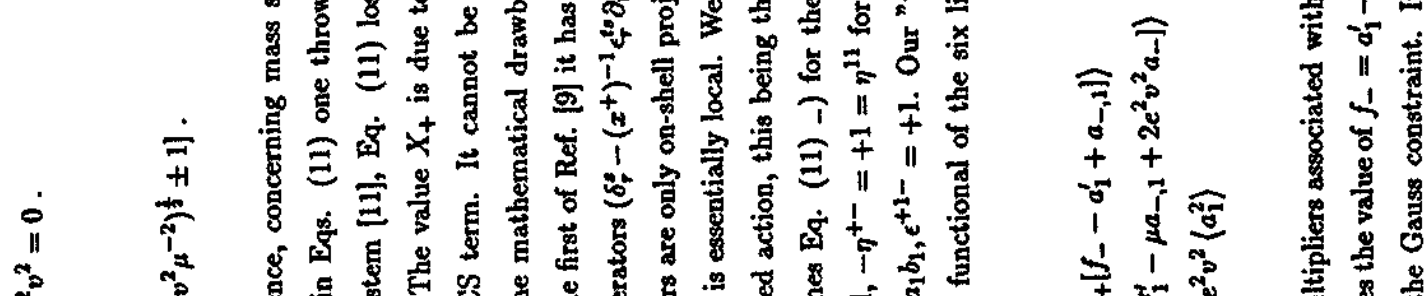

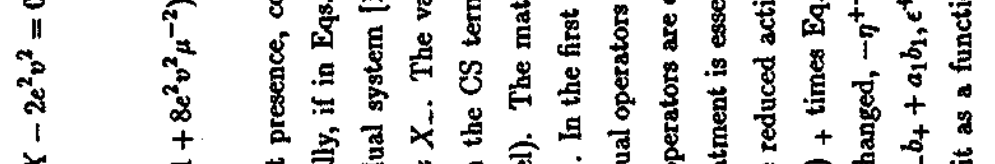

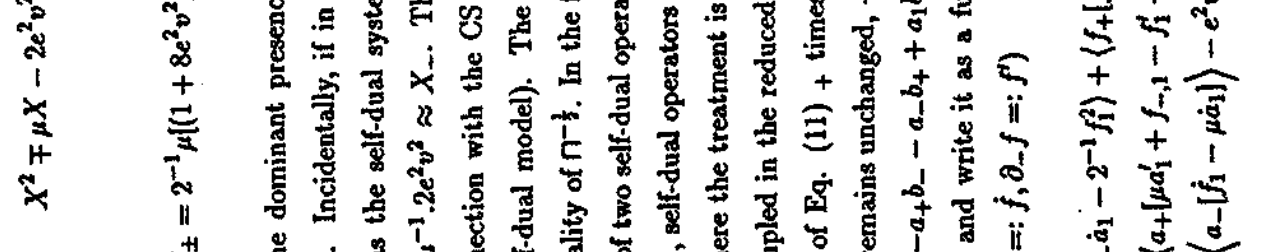

*

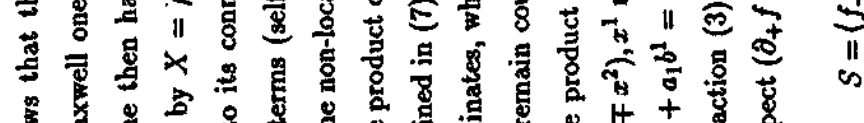

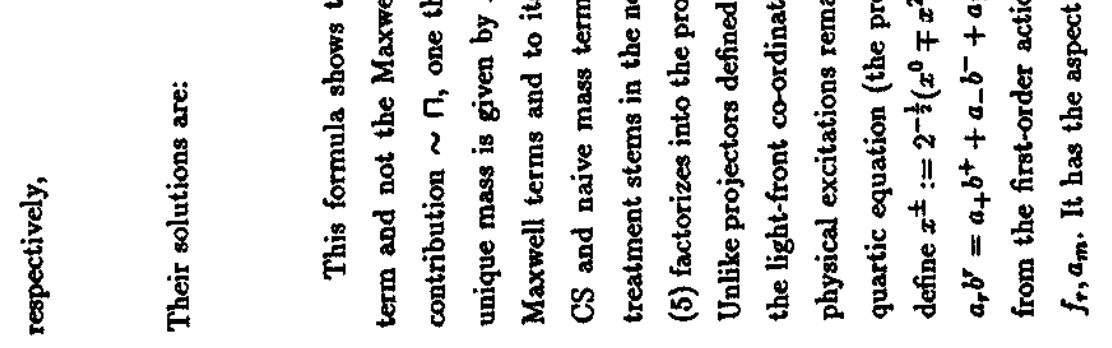

i

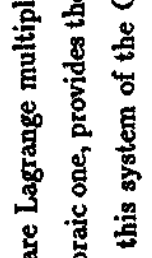

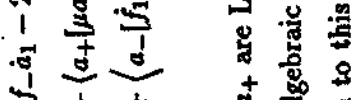

喜魯量

$+\stackrel{5}{*}$

嘉喜

政

害嘉

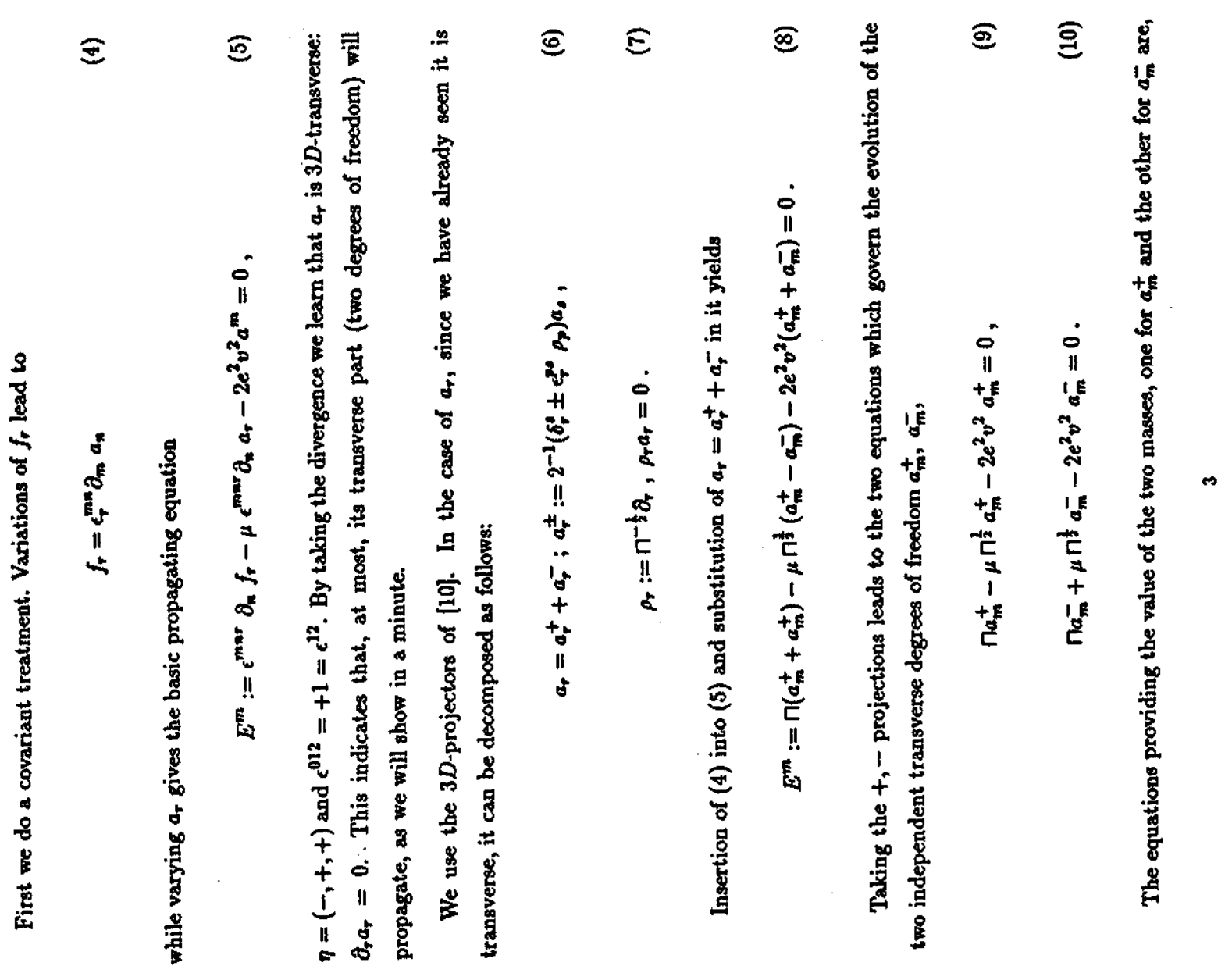



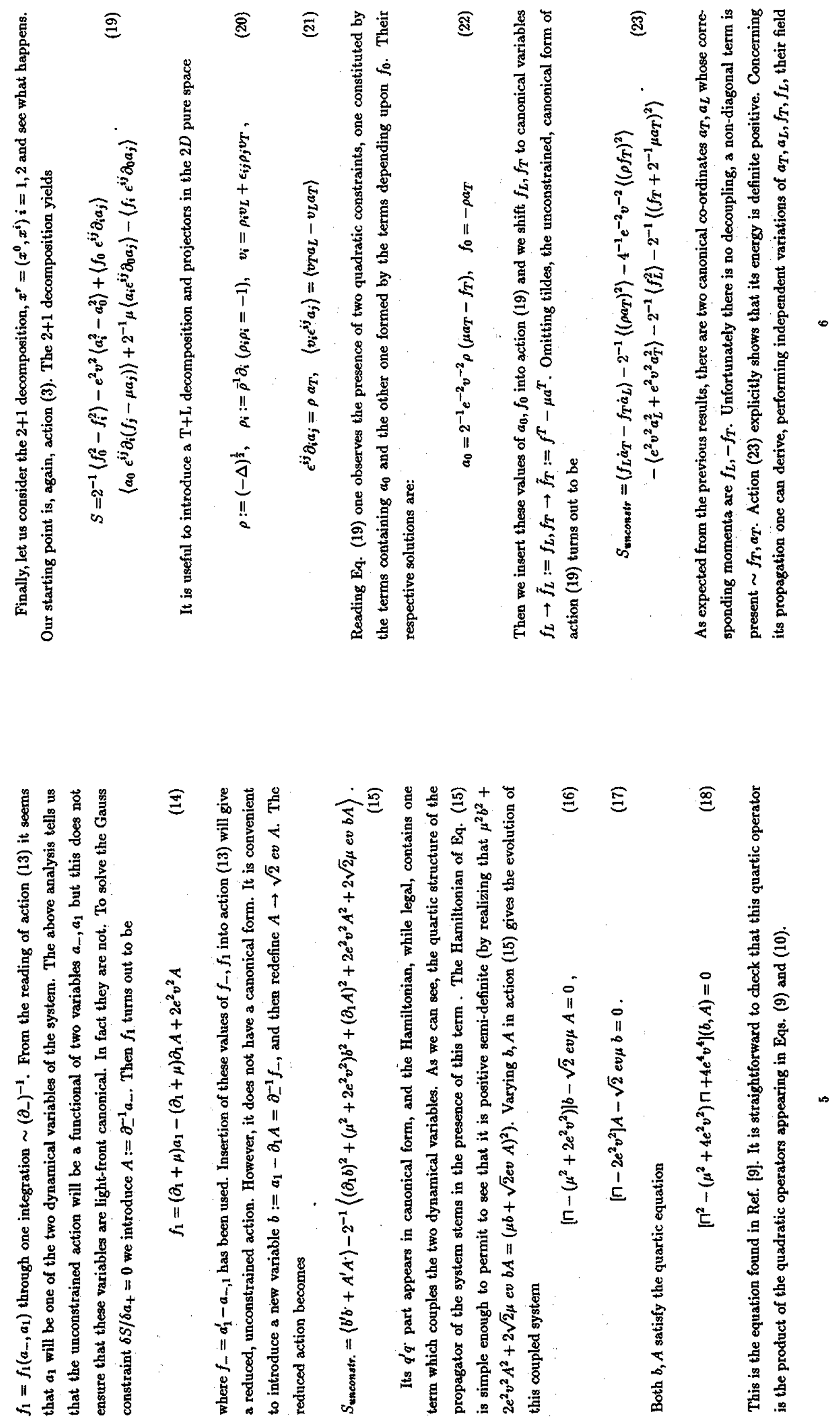

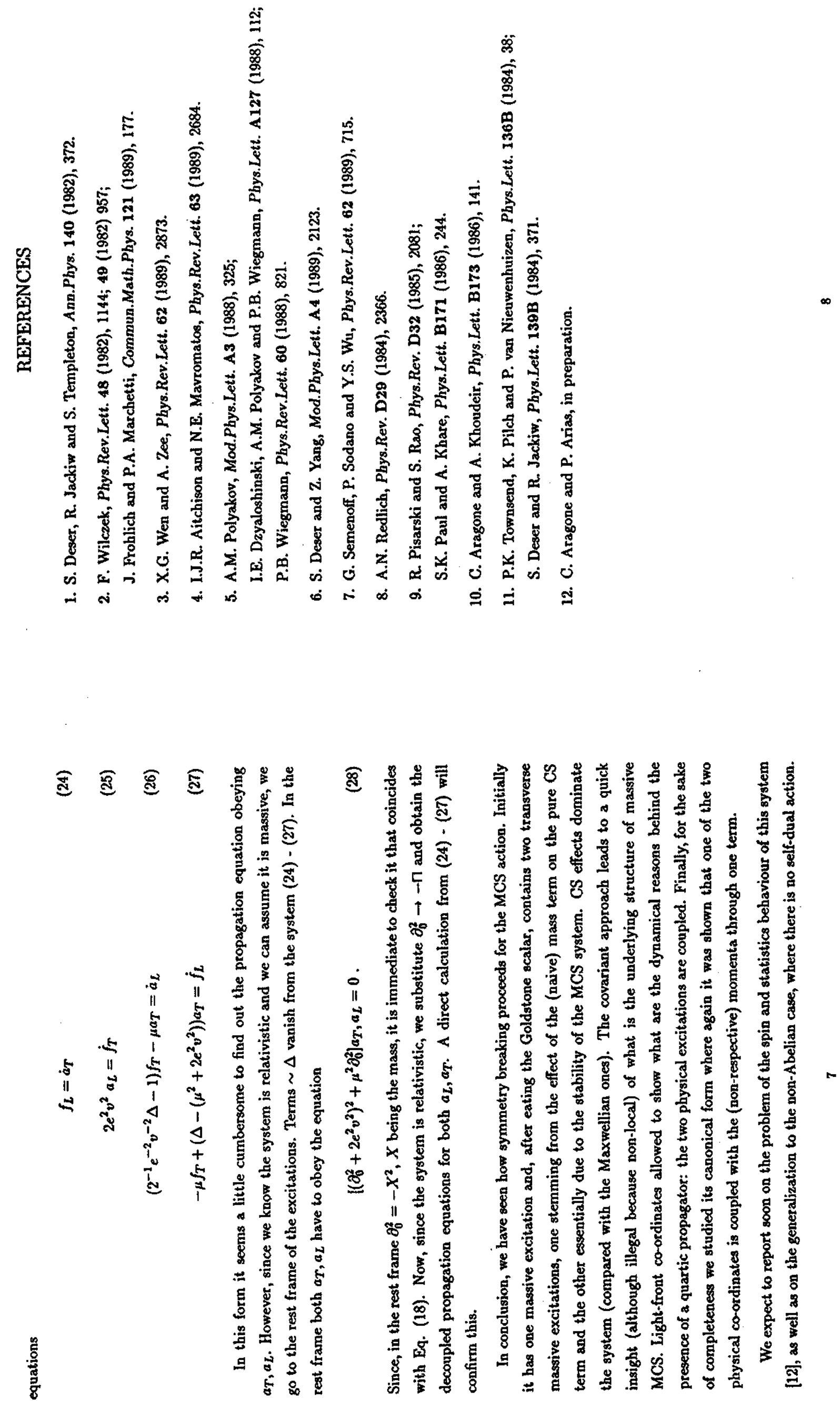